\title{
Laryngeal function reconstruction with hyoid osteomuscular flap in partial laryngectomy for laryngeal cancer
}

\author{
BOJUN WEI, HONG SHEN and HONG XIE \\ Department of Otorhinolaryngology Head and Neck Surgery, Beijing Shijitan Hospital, \\ Capital Medical University, Beijing 100038, P.R. China
}

Received July 4, 2014; Accepted March 12, 2015

DOI: $10.3892 / \mathrm{ol} .2015 .3362$

\begin{abstract}
The present study aimed to evaluate the clinical outcome of using a hyoid osteomuscular flap to repair the laryngeal defect after extended vertical partial laryngectomy. A total of 26 glottic cancer patients underwent reconstruction with osteomuscular hyoid flaps following tumor resections. Ipsilateral arytenoid cartilage was resected in all cases, and the upper region of the cricoid cartilage was resected in 11 cases. Selective ipsilateral level II, III and IV neck dissections were performed in node $(\mathrm{N})$-positive patients and ipsilateral level II, and III neck dissections in N0 patients. The bone grafts were then fixed to the cricoid and contralateral thyroid cartilages. Invasion of the thyroid cartilage endochorium was present in 12 cases and lymph nodes metastases was present in 11 cases. The extubation rate of the tracheostomy tube was $100 \%$. The glottides of all patients were almost symmetrical. Patients were followed up for 2-7 years. One patient developed local recurrence, ipsilateral regional recurrence, contralateral regional recurrence and lung metastasis, respectively. The disease-free survival rates at 3 and 5 years were 100\% (20/20) and 79\% (11/14), respectively. Overall, laryngeal function recovered well upon hyoid osteomuscular flap reconstruction following extended vertical partial laryngectomy, with a high extubation rate and good sound quality.
\end{abstract}

\section{Introduction}

Cancer of larynx was the second most common cancer in head and neck following oral cavity cancer, as well as the second most frequently cancer occurred in respiratory tract

Correspondence to: Dr Bojun Wei, Department of Otorhinolaryngology Head and Neck Surgery, Beijing Shijitan Hospital, Capital Medical University, 10 Tieyilu Yangfangdian, Haidian, Beijing 100038, P.R. China

E-mail: weibojun1015@sina.com

Key words: extended vertical partial laryngectomy, laryngeal cancer, hyoid osteomuscular flap except lung cancer. According to GLOBOCAN 2012, there were an estimate of 156,877 new cases and 83,376 deaths in the world, and the adjusted incidence and mortality rates were 2.1/100,000 and 1.1/100,000, respectively (1). For patients with laryngeal cancer, surgical treatment provides the best therapeutic effects. Even with a partial laryngectomy, patients may recover at least a certain degree of laryngeal function, and return to society and enjoy life almost immediately after treatment. Direct laryngoscopy must be performed prior to the aryepiglottoplasty to decide what amount of tissue to resect. Endoscopic aryepiglottoplasty with use of microlaryngeal instruments is an effective and safe method of the treatment of severe form of laryngomalacia. It is better to perform this procedure in general anesthesia without intubation (2)

Concurrent chemoradiotherapy has been applied to treat patients with stage III and IV oropharyngeal, hypopharyngeal or laryngeal cancer. Notably, based on clinical outcomes, certain chemotherapy and radiotherapy practitioners have even suggested that concurrent chemoradiotherapy may replace surgery to treat laryngeal cancer (3). This is contrary to the viewpoint of the majority of otolaryngologists, as sole treatment by chemoradiology has not provided superior clinical outcomes to laryngeal cancer patients in the past decades $(4,5)$.

The arytenoids, aryepiglottic folds and epiglottis work together to close the laryngeal inlet during swallowing, so as to prevent aspiration (6). Following the resection of arytenoid cartilage, the glottis cannot close completely without proper reconstruction. Head and neck surgeons should carefully consider the preservation of laryngeal function, particularly in patients with late-stage laryngeal cancer. In the present study, a novel reconstruction technique for arytenoid region defects is investigated, which not only avoid the complications of aspiration and laryngeal stenosis, but also preserves sound quality post-recovery.

\section{Materials and methods}

Patients. A total of 26 laryngeal cancer patients, 25 males and 1 female, from the Beijing Shijitan Hospital (Capital Medical University, Beijing, China) were included in the present study. The age of the cohort ranged between 44 and 78 years old, with a median age of 61 years old. All cases were diagnosed as glottic carcinoma, with 9 cases staged as T3N1aM0, 4 cases as T3N2bM0, 7 cases as T3N0M0 and 3 cases as T2N0M0. 
Additionally, 3 cases encountered recurrent diseases following initial treatment in other hospitals. Pre-operative laryngeal endoscopy showed vocal cord fixation in 23 cases, including 3 cases of arytenoid area recurrence after initial therapy, and impaired vocal cord movement in another 3 cases. The vocal cartilage or arytenoid area was involved in all cases, with anterior commissure involvement in 15 patients and tumors approaching the posterior commissure in 3 patients. None of the patients exhibited contralateral vocal cord involvement. : The study was approved by the ethics committee of Capital Medical University (Beijing, China).

Treatment. While 3 patients with tumors approaching the posterior commissure were treated with $50 \mathrm{~Gy}$ pre-operative radiation followed by surgery in 2-3 weeks, the remaining 23 patients were managed by extended vertical partial laryngectomy without radiation or chemotherapy.

Surgical procedure. The surgical procedure consisted of the following: i) Intubation and general anesthesia were performed following conventional tracheotomy. ii) The surgical incision was designed according to the tumor $(\mathrm{T})$ and node $(\mathrm{N})$ classification, and skin striae. The flap was lifted to expose the surgical area. Selective neck dissections of ipsilateral level II, III and IV sections were performed for N-positive patients and ipsilateral I and III neck dissections were performed for N0 patients. The ipsilateral superior thyroid artery and vein, and the superior laryngeal artery and its branches into strap muscles were preserved (Fig. 1). iii) The ipsilateral arytenoid cartilage, vocal cords, laryngeal ventricle, ventricular fold and anterior two-thirds of the thyroid cartilage were resected (Fig. 2). If the anterior commissure was involved, the anterior regions of the contralateral vocal cord and ventricular fold were removed. Surgical margins were confirmed upon frozen sections. iv) The lingual artery and hypoglossal nerve were then secured. The supra-hyoid muscle group was cut off and a section of the hyoid bone was intercepted as the graft. The bone graft was flipped anteriorly downwards with the strap muscles to the laryngeal space. The fascia of the strap muscles, the external perichondrium of the thyroid cartilage and the thyrohyoid membrane were all preserved. v) The bone graft was then tailored by measuring the defect and thinned to meet the thickness of the thyroid cartilage. If the cartilage of the anterior commissure was resected, the bone graft was carved into an inverted $\mathrm{V}$ shape so that the anteroposterior diameter of the glottis could be restored. vi) The posterolateral end of the bone graft was placed at the defective site of the arytenoid cartilage and sutured to the adjacent soft tissue. It was ensured that the inside edge of the bone graft did not exceed the midline and that the muscle pedicle did not exceed the inside edge of the bone graft. The posterior end of the bone graft was covered by hypopharyngeal mucosa and sutured with the posterior commissure. vii) Next, the anterior end of the bone graft was fixed to the edge of the contralateral thyroid cartilage, ensuring the correct alignment of membrane to membrane and bone to cartilage, and confirming that the end of the bone graft was not exposed. viii) The pharynx was then closed, a drainage tube was placed and the incision was closed.

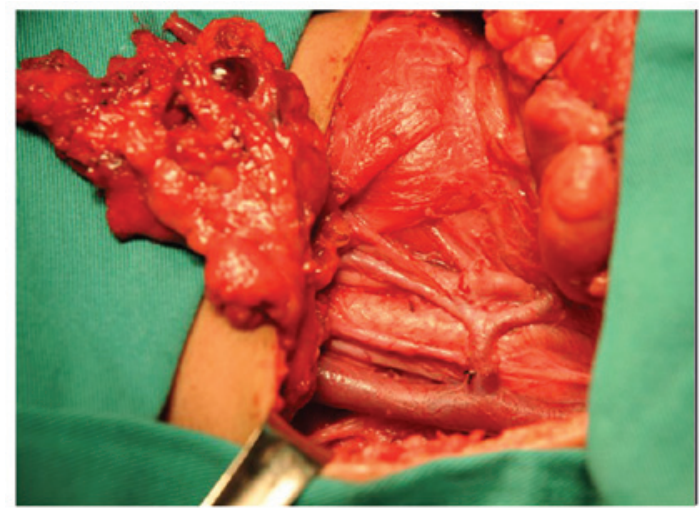

Figure 1. Superior thyroid artery and vein, and superior laryngeal artery and its branches into the strap muscles were preserved.

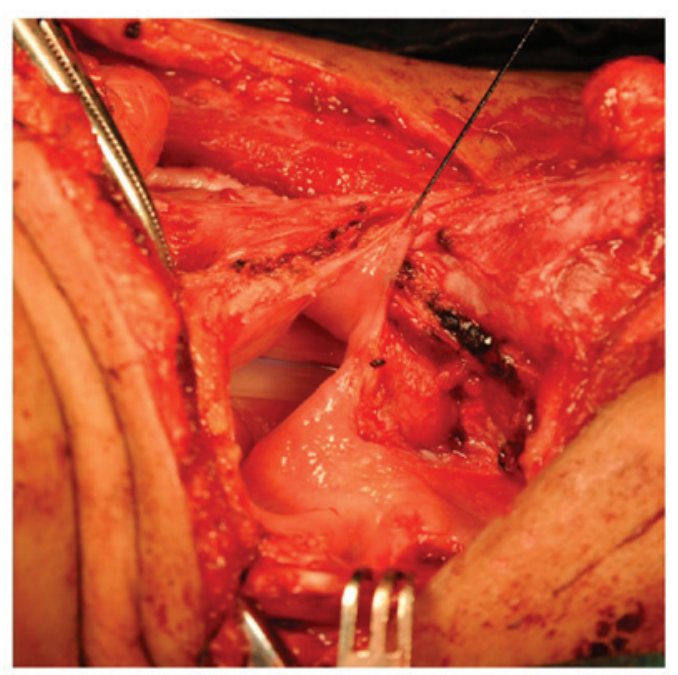

Figure 2. Ipsilateral arytenoid cartilage, vocal cord, laryngeal ventricle, ventricular fold and anterior two-thirds of the thyroid cartilage were resected.

Post-operative treatment. Conventional tracheotomy and continuous wound drainage were performed. Drainage tubes were removed once the drainage fluid level reached $<15 \mathrm{ml}$ over $24 \mathrm{~h}$. Nasogastric feeding was applied on the first post-operative day. Patients were provided with oral intake on the seventh post-operative day and nasal feeding tubes were removed if no evident aspiration was observed. The tracheotomy tubes were plugged if no dyspnea was observed. Endoscopic evaluations were then applied after one month. If no granulation was found, the tracheal tubes were then removed. If granulation was present, this was removed by a second surgery and the patients were kept under observation for an additional month. All patients were periodically reviewed by laryngeal endoscopy, ultrasound of the neck and computed tomography of the throat and chest.

\section{Results}

Pathological examination showed invasion of the thyroid cartilage perichondrium in 12 cases and lymph nodes metastases in 11 cases. Negative surgical margins were achieved in all cases. Nasal feeding tubes were removed from 19 patients within 10 days post-surgery and in 6 patients within 3 weeks. A 


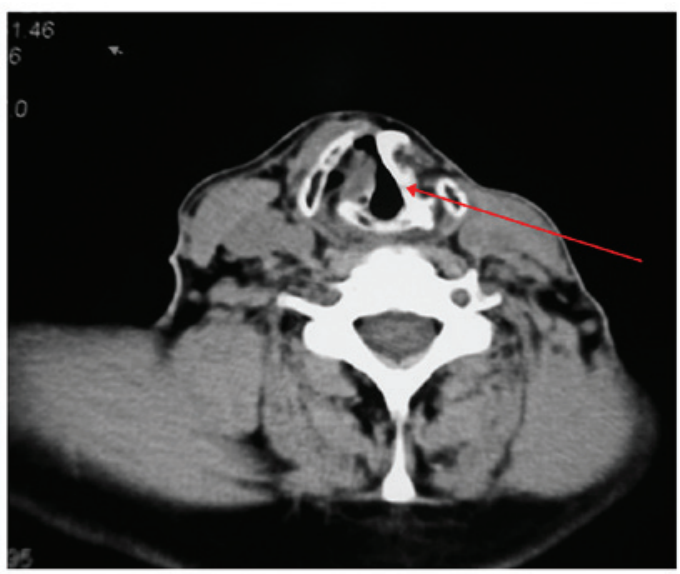

Figure 3. Axial computed tomography scan of the reconstructed larynx showing the symmetrical glottic region. The arrow indicates the hyoid.

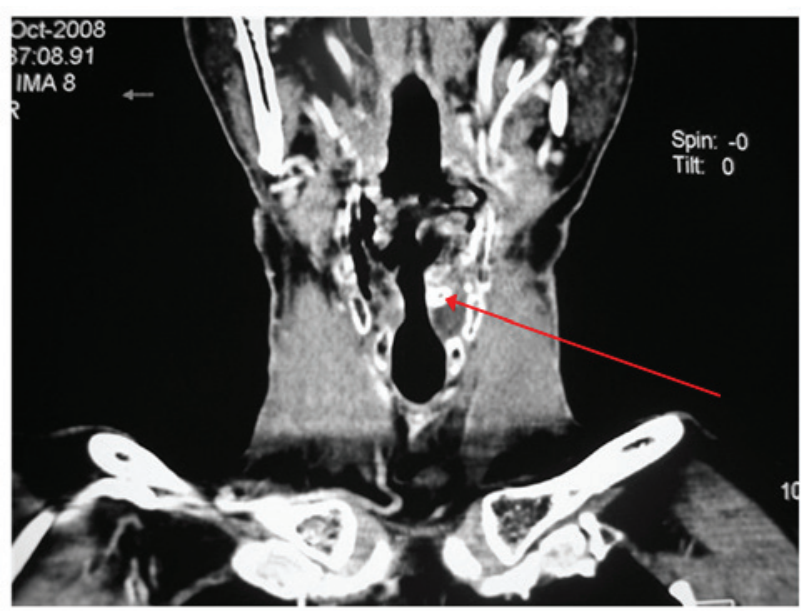

Figure 4. Coronal computed tomography scan of the reconstructive larynx showing the symmetrical glottic region. The arrow indicates the hyoid.
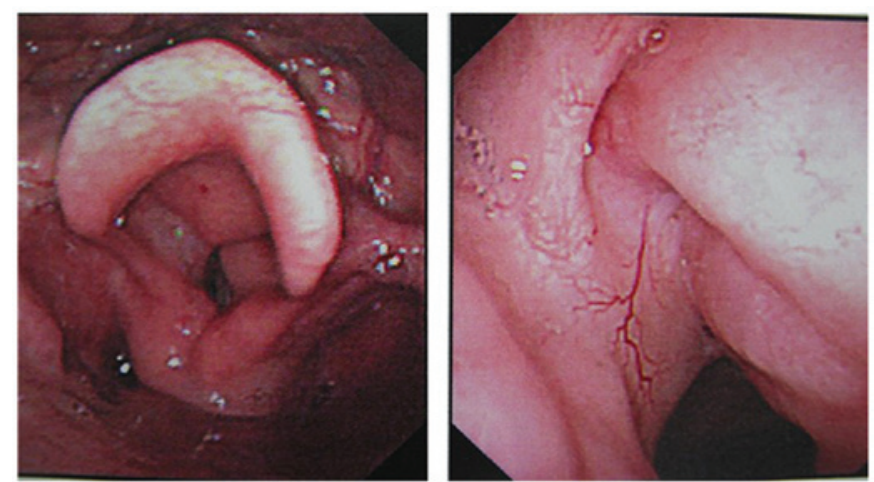

Figure 5. Symmetrical glottides were able to open (left) and close (right) well.

pharyngeal fistula was recorded in one patient; recovery from this complication was observed following 3 weeks of conservative treatment, consisting of drainage and cleaning the fistula. The extubation rate of the tracheostomy tube was $100 \%$. In total, 18 patients were extubated within 1 month post-surgery and 6 patients within 2 months. In addition, 2 patients underwent secondary surgical procedures to remove granulation tissue of the anterior commissure, and were then observed for 1 month to confirm no granulation prior to extubation. No aspiration pneumonia or throat adhesions were observed. The glottides of all patients were almost symmetrical (Figs. 3 and 4), and were able to open fully and close well (Fig. 5). Follow-up visits were scheduled after 2-7 years. One patient developed local recurrence, ipsilateral regional recurrence, contralateral regional recurrence and lung metastasis, respectively. The 3-year disease-free survival rate was $100 \%(20 / 20)$ and the 5-year disease-free survival rate was $79 \%(11 / 14)$.

\section{Discussion}

The complications of partial laryngectomy include laryngeal stenosis, aspiration and poor pronunciation $(7,8)$. The removal of arytenoid cartilage increases the incidence and grade of complications. Serious aspiration not only affects the patient's quality of life, but also increases the risk of patient mortality. Patients with serious aspiration often require surgical repair of the non-functioning glottis, with certain patients being required to undergo a total laryngectomy. Therefore, the repair of the laryngeal defect following the removal of the arytenoid cartilage and the minimization of post-operative aspiration is a focus of attention. Although a high decannulation rate may be obtained without repair, a severe hoarseness would result from this. Thus, it has been suggested that no reconstruction of arytenoid area defects should be conducted at all (9). Single or double pedicle sternohyoid muscle flaps are therefore a good option for surgery (10). Clinicians have previously successfully restored the arytenoid defect with pedicle hyoid bone and improved the reconstruction quality, with a post-operative extubation rate of up to $87.9 \%$ (11). Notably, the grafted muscle tissues in the glottic area have a different degree of atrophy after reconstruction, and severe atrophy of the muscle could lead to marked hoarseness, while inadequate atrophy may affect extubation. It is therefore more difficult to determine the ideal thickness of the muscle flap. Moreover, the preserved contralateral thyroid cartilage will be displaced anterioposteriorly following the loss of support from the other half, thus shortening the anteroposterior diameter of the glottis. The supracricoid subtotal laryngectomy has simple steps and a high extubation rate, but provides poor sound quality $(12,13)$. Extensive rehabilitation following surgery is also commonly required in supracricoid subtotal laryngectomy patients.

The use of a hyoid flap repairs defects in the glottis and arytenoid areas, and restores the laryngeal frame and anteroposterior diameter. The technique also has additional advantages, including reducing aspiration, as the thickness of hyoid is suitable for defects of the arytenoid area and the structure of hypopharynx can be restored, epiglottis functionally preserved, and pharynx and larynx successfully separated during swallowing. If there is huge defect of the cricoid, it can be compensated for by a corresponding increase in the amount of muscle attached to the hyoid. Therefore, post-operative aspiration develops with a lower occurrence rate, less severity and a quick patient recovery.

The hyoid bone is large enough to restore different types of laryngeal frame defects. If the thyroid cartilage at the anterior commissure and the anterior region of the contralateral vocal 
cord is removed, the diameter of the glottis can be restored by carving the hyoid bone into an inverted $\mathrm{V}$ shape. It also prevents the deflection of the glottis following reconstruction with soft tissue alone. There is enough periosteum of hyoid to be used for fixation. There is no displacement and hardly any atrophy of the bone graft after fixation. Thus, the diameter of the glottis is restored well and in a long-standing manner. The interior tissue of the hyoid is ligament-like membrane that can be easily sutured and epithelialized. All these are favorable factors for the prevention of laryngeal stenosis and an increase in the extubation rate.

In the present study, the post-operative voices of 6 patients were significantly improved, while 13 patients had nearly the same quality of voice pre- and post-operatively. Hoarseness was aggravated post-operatively in seven patients, but their daily communication was not affected. The 3 cases that underwent pre-operative radiotherapy were successfully reconstructed.

In summary, the present study demonstrated that a hyoid osteomuscular flap characterized by hard bone and soft muscle tissue can be applied to achieve restoration of laryngeal function and structure. This technique is suitable for the reconstruction after partial laryngectomy in patients with laryngeal cancer.

\section{References}

1. van Dijk BA, Karim-Kos HE, Coebergh JW, Marres HA and de Vries E: Progress against laryngeal cancer in The Netherlands between 1989 and 2010. Int J Cancer 134: 674-681, 2014.
2. Pucher B and Grzegorowski M: Surgical treatment of laryngomalacia in children. Otolaryngol Pol 60: 349-354, 2006 (Article in Polish).

3. Forastiere AA, Goepfert H, Maor M, et al: Concurrent chemotherapy and radiotherapy for organ preservation in advanced laryngeal cancer. N Engl J Med 349: 2091-2098, 2003.

4. Hoffman HT, Porter K, Karnell LH, et al: Laryngeal cancer in the United States: changes in demographics, patterns of care and survival. Laryngoscope 116 (Suppl 111): 1-13, 2006.

5. Silver CE, Beitler JJ, Shaha AR, et al: Current trends in initial management of laryngeal cancer: the declining use of open surgery. Eur Arch Otorhinolaryngol 266: 1333-1352, 2009.

6. Liu B, Pan Z and Ji W: Reconstruction of laryngeal defect in vertical partial laryngectomy with resection of arytenoid cartilage. Zhonghua Er Bi Yan Hou Tou Jing Wai Ke Za Zhi 40: 52-55, 2005 (In Chinese).

7. Ulualp SO: Mapping regional laryngopharyngeal mechanoreceptor response. Clin Exp Otorhinolaryngol 7: 319-323, 2014.

8. Ouyang D1, Liu TR, Liu XW, Chen YF, Wang J, Su X and Yang AK: Combined hyoid bone flap in laryngeal reconstruction after extensive partial laryngectomy for laryngeal cancer. Eur Arch Otorhinolaryngol 270: 1455-1462, 2013.

9. Diab S, Pascoe J, Shahriar M, Read D, Kinde H, Moore J, Odani J and Uzal F: Study of laryngopharyngeal pathology in Thoroughbred horses in southern california. Equine Vet J 41: 903-907, 2009.

10. Jia S, Sun B and Shang L: Extended vertical partial laryngectomy for treatment of the glottic cancer with T3 category. Zhonghua Er Bi Yan Hou Ke Za Zhi 31: 365-367, 1996 (In Chinese).

11. Tu G, Tang P and He Y: The use of hyoid osteomuscular flap in extended partial laryngectomy. Zhonghua Er Bi Yan Hou Ke Za Zhi 31: 39-42, 1996 (In Chinese).

12. Yan D, Zhang B, Qi Y, et al: Supracricoid partial laryngectomy versus other traditional partial laryngectomy for selected laryngeal cancers. Lin Chung Er Bi Yan Hou Tou Jing Wai Ke Za Zhi 24: 828-831, 2010 (In Chinese).

13. Zhang SY, Lu ZM, Chen LS, et al: Supracricoid partial laryngectomy cricohyoidoepiglottopexy (SCPL-CHEP) versus vertical partial laryngectomy for the treatment of glottic carcinoma. Eur Arch Otorhinolaryngol 270: 1027-1034, 2013. 